\title{
OLYMPUS and the Proton Form Factor Puzzle
}

\author{
Lauren Ice and Ricardo Alarcon \\ for the OLYMPUS collaboration
}

Department of Physics

Arizona State University

Tempe, Arizona 85287

\begin{abstract}
Recent measurements of the proton electric to magnetic form-factor ratio using polarization techniques reveal a large discrepancy with measurements found using the Rosenbluth separation technique. It has been proposed that this discrepancy is due to non-negligible multiple photon exchange contribution to the electron-proton elastic scattering cross section. The OLYMPUS experiment will measure the multiple photon exchange contribution by finding the cross section ratio of positron-proton to electron-proton scattering within $1 \%$. The experiment will be carried out at DESY laboratory in Hamburg Germany using the electron and positron storage ring DORIS and an internal unpolarized hydrogen gas target. The scattered particles will be detected using the Bates Large Acceptance Spectrometer Toroid (BLAST).
\end{abstract}

Keywords: Proton elastic form factors, two-photon exchange

PACS: $13.40 . \mathrm{Gp}, 13.60 .-\mathrm{r}, 14.20 . \mathrm{Dh}$

\section{INTRODUCTION}

The OLYMPUS experiment aims at measuring the cross section ratio of positron-proton to electron-proton elastic scattering, $\frac{\sigma_{e^{+}} p}{\sigma_{e^{-}}}$, as a confirmation of a non-negligible multiple photon exchange contribution to electron-proton scattering. The motivation for this experiment came from a deviation from unity of the electric to magnetic form factor ratio of the proton in polarization transfer experiments in contrast to measurements taken using the Rosenbluth separation technique. Since the Rosenbluth formula assumes a one virtual photon exchange between the electron and proton, higher order radiative corrections, in particular the multiple photon exchange, is the expected culprit for this discrepancy. [1]

Under the Born approximation (the one photon exchange) the cross sections for electron-proton and positron-proton elastic scattering are identical giving $\frac{\sigma_{e^{+}}}{\sigma_{e^{-}}}=1$. When taking in higher order radiative corrections into consideration, the electron-proton cross section contains an interference term between the one and two photon exchange amplitudes that is reversed for positron-proton elastic scattering. It is predicted that this interference term will cause there to be a deviation in $\frac{\sigma_{e^{+}} p}{\sigma_{e^{-}} p}$ from unity by about $6 \%$.

OLYMPUS will measure $\frac{\sigma_{e^{+} p}}{\sigma_{e^{-}} p}$ to $1 \%$ for a momentum transfer range of $0.6<\mathrm{Q}^{2}<$ $2.2(\mathrm{GeV} / \mathrm{c})^{2}$. The experiment will take place at the Deutsches Elektronen-Synchrotron (DESY) laboratory using the DORIS ring. The DORIS ring is a multi-GeV positron and electron storage ring with maximum energy of $4.5 \mathrm{GeV}$ and electron current of 100 
mA. OLYMPUS will be running at an energy of $2.0 \mathrm{GeV}$ with an unpolarized internal hydrogen gas target. The cross section measurement will be made with the (BLAST) detector. [2]

\section{NUCLEON FORM-FACTORS}

The electric, $G_{E p}$, and magnetic, $G_{M p}$, form factors of the proton are observables that characterize the electromagnetic structure of the proton in the non-perturbative region. Most generally, they are Fourier transforms of the spacial charge and current densities of the proton [3] and are crucial to our understanding of quantum chromodynamics, nucleon-nucleon interactions, the proton charge radius, the Lamb shift and tests of the weak interaction at low $\mathrm{Q}^{2}$. [4] The electric and magnetic form factors can be written as functions of the Pauli, $F_{1 p}\left(Q^{2}\right)$ and Dirac, $F_{2 p}\left(Q^{2}\right)$ Sachs form factors as [5]

$$
G_{E p}\left(Q^{2}\right)=F_{1 p}\left(Q^{2}\right)-\tau \kappa_{p} F_{2 p}\left(Q^{2}\right)
$$

and

$$
G_{M p}\left(Q^{2}\right)=F_{1 p}\left(Q^{2}\right)+\kappa_{p} F_{2 p}\left(Q^{2}\right)
$$

where $\tau=\frac{Q^{2}}{4 M^{2}}, Q^{2}=-q^{2}$ and $\kappa_{p}$ is the nucleon magnetic moment. The Pauli and Dirac Sachs form factors are defined in the following way [4]

$$
\left\langle N\left(P^{\prime}\right)\left|J_{E M}^{\mu}(0)\right| N(P)\right\rangle=\bar{u}\left(P^{\prime}\right)\left[\gamma^{\mu} F_{1 p}^{N}\left(Q^{2}\right)+i \sigma^{\mu v} \frac{q_{v}}{2 M} F_{2 p}^{N}\left(Q^{2}\right)\right] u(P)
$$

where $J_{E M}^{\mu}$ is the electromagnetic current, $\sigma^{\mu v}=\frac{i}{2}\left[\gamma^{\mu}, \gamma^{v}\right]$ and $q_{v}=\left(P^{\prime}-P\right)_{v}$ and $\bar{u}\left(P^{\prime}\right)$ and $u(P)$ are Dirac spinors. The electric and magnetic form factors are found experimentally using electron-proton scattering. Electron-proton scattering is ideal for measuring the form factors of the proton since it allows for the proton structure to be probed using electromagnetic interactions with minimal perturbation of the proton structure.

\section{Rosenbluth Separation Technique}

The method historically used to measure the electric and magnetic form factors of the proton is the Rosenbluth Separation Technique. [6] In this technique the differential cross section for electron-proton scattering is measured over a range of electron scattering angles $\theta$ for fixed $\tau=\frac{Q^{2}}{4 M_{p}^{2}}$. The data is then analyzed using the Rosenbluth cross section formula. [3]

$$
\frac{d \sigma}{d \Omega}=\left(\frac{d \sigma}{d \Omega}\right)_{M o t t} \frac{\varepsilon G_{E p}^{2}\left(Q^{2}\right)+\tau G_{M p}^{2}\left(Q^{2}\right)}{\varepsilon(1+\tau)}
$$

where $\varepsilon$ is the virtual photon polarization given by:

$$
\varepsilon=\frac{1}{1+2(1+\tau) \tan ^{2} \theta / 2}
$$


and

$$
\left(\frac{d \sigma}{d \Omega}\right)_{M o t t}=\frac{\alpha^{2}}{4 E^{2}} \frac{\cos ^{2} \theta / 2}{\sin ^{4} \theta / 2} \frac{E^{\prime}}{E}
$$

is the Mott scattering cross section for electron-proton scattering. The Mott formula assumes that the electron and proton only interact through the exchange of one virtual photon, and that the proton is an infinitely massive, spinless point charge that does not recoil. The Rosenbluth extends the Mott formula to include the charge and current distributions of the proton. [3]

By using the Rosenbluth separation technique the electromagnetic form factors can be measured. When this is done, it is found that the electric and magnetic form factors are both proportional to the dipole form factor $G_{D}=\left(1+\frac{Q^{2}}{0.71}\right)^{-2}$.[5] This is the Fourier transform of $\rho_{D}(r)=\rho_{0} e^{-\sqrt{0.71} r}$, a smoothly decaying exponential function. Therefore the ratio of the electric to magnetic form factors as a function of $\mathrm{Q}^{2}$ is just a constant, $\frac{1}{\mu_{p}}$, where $\mu_{p}$ is the magnetic moment of the proton.

$$
\frac{\mu_{p} G_{E p}\left(Q^{2}\right)}{G_{M p}\left(Q^{2}\right)}=1
$$

\section{Polarization Transfer Methods}

Another method for measuring the electric and magnetic form factors is with polarization techniques by measuring the polarization of the recoil protons in $\vec{e} p \rightarrow e \vec{p}$ scattering or the beam-target polarization asymmetry in $\vec{e} \vec{p} \rightarrow e p$ scattering. [4] The first of these techniques is called the polarization transfer method. In this method a beam of polarized electrons is scattered off of an unpolarized proton target. The polarization of the recoiled protons is measured with a polarimeter giving the longitudinal polarization, $P_{l}$, and the perpendicular polarization, $P_{t}$. These polarizations can be written in terms of the electric and magnetic form-factors as [7]

$$
I_{0} P_{t}=-2 \sqrt{\tau(1+\tau)} G_{E p} G_{M p} \tan ^{2} \theta / 2
$$

and

$$
I_{0} P_{l}=\frac{1}{M}\left(E+E^{\prime}\right) \sqrt{\tau(1+\tau)} G_{M p}^{2} \tan ^{2} \theta / 2
$$

where $I_{0}=G_{E p}^{2}+\frac{\tau}{\varepsilon} G_{M p}^{2}$. From these equations the electric to magnetic form factor ratio can be found as

$$
\frac{G_{E p}}{G_{M p}}=-\frac{P_{t}}{P_{l}} \frac{\left(E+E^{\prime}\right)}{2 M} \tan ^{2} \theta / 2
$$

When this experiment was carried out at Jefferson Laboratory it was found that the electric to magnetic form factor ratio, instead of being unity as measured with the Rosenbluth separation technique, was actually decreasing significantly with $Q^{2}$. [7] The world's data for electric to magnetic form factor ratio of the proton as measured with the Rosenbluth separation technique and polarization transfer is shown in Figure 1. 


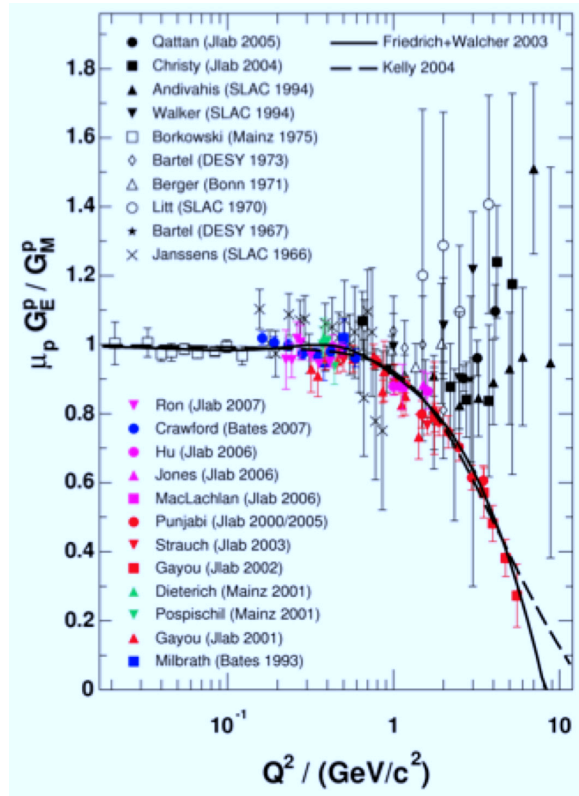

FIGURE 1: World Data for the electric to magnetic form-factor ratio: The black symbols are from measurements using the Rosenbluth separation technique and the colored symbols are from polarization measurements including the polarization measurements made at JLab.[2]

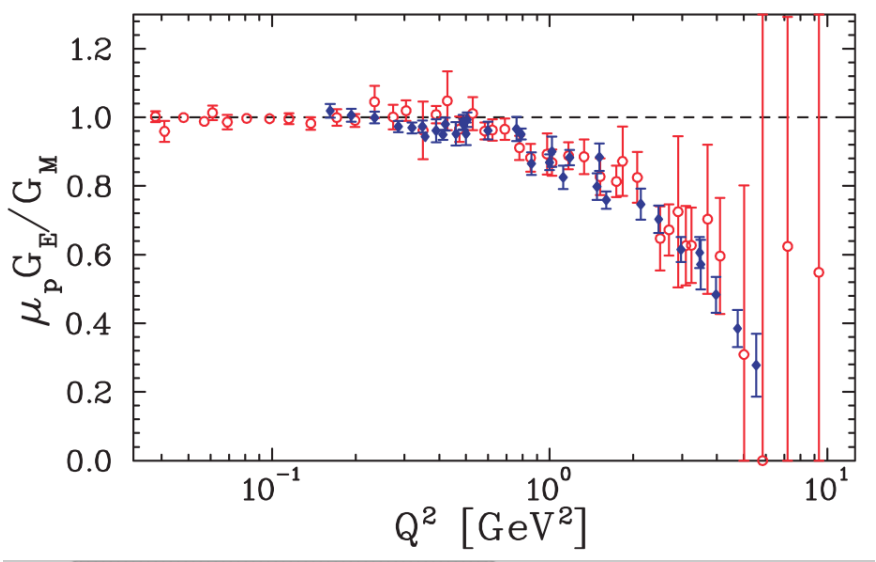

FIGURE 2: The electric to magnetic form factor ratio from Rosenbluth separation technique as analyzed with two-photon exchange (red) and data from polarization transfer method (blue).[9]

\section{MULTIPLE PHOTON EXCHANGE}

Since the Rosenbluth formula assumes only one virtual photon exchange a probable explanation for the discrepancy is that higher order radiative corrections in electron-proton scattering, in particular the two photon exchange amplitudes, need to be taken into consideration. [8] When two-photon exchange is taken into account with the Rosenbluth technique it is found that the electric to magnetic form factor ratio follows the decreasing trend as found with the polarization transfer method. This is shown in Figure 2. [9]

The scattering amplitude $M$ for electron-proton scattering can be written out as a QED perturbative expansion with expansion parameter $\alpha=\frac{e^{2}}{4 \pi \varepsilon_{0} \hbar c} \approx \frac{1}{137}$, the fine structure constant. [10] In this expansion the first order term is the one photon exchange where as the second order terms have two virtual photons and include the exchange of two 


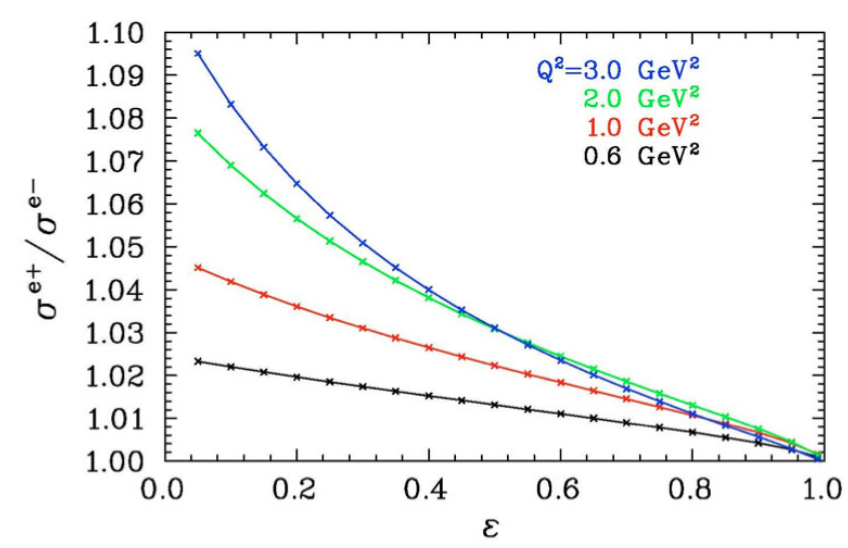

FIGURE 3: Theoretical values for the positron-proton to electron-proton elastic scattering cross section ratio for various $Q^{2}$ as a function of virtual polarization $\varepsilon$. [2]

"hard" photons. Symbolically, this is $M=M_{1 \gamma}+M_{2 \gamma}+\ldots$. The one photon exchange amplitude is identical for positron-proton scattering and electron-proton scattering, but the two-photon exchange amplitude is reversed. When the cross section is found as the modulus square of the scattering amplitude, there remains an interference term between the one and two photon exchange amplitudes that is of opposite sign for electron-proton and positron-proton scattering. [11]

$$
\begin{aligned}
\frac{d \sigma_{e^{-} p}}{d \Omega} & =\left|M_{1 \gamma}-M_{2 \gamma}+\ldots\right|^{2} \\
& =\left|M_{1 \gamma}\right|^{2}-2 \operatorname{Re} M_{1 \gamma} M_{2 \gamma}+\ldots \\
\frac{d \sigma_{e^{+} p}}{d \Omega} & =\left|M_{1 \gamma}+M_{2 \gamma}+\ldots\right|^{2} \\
& =\left|M_{1 \gamma}\right|^{2}+2 \operatorname{Re} M_{1 \gamma} M_{2 \gamma}+\ldots
\end{aligned}
$$

Since the interference term is proportional to $\alpha^{3}$ it causes there to be a measurable deviation from unity in $\frac{\sigma_{e^{+}}}{\sigma_{e^{-}}}$. By measuring $\frac{\sigma_{e^{+} p}}{\sigma_{e^{-}}}$with high precision this multiple photon exchange amplitude can be found.

\section{PROPOSED EXPERIMENT}

The OLYMPUS experiment will precisely measure $\frac{\sigma_{e^{+} p}}{\sigma_{e^{-}}}$as a function of the virtual photon polarization, $\varepsilon$ to within $1 \%$ in order to find the multiple photon exchange contribution to the electron-proton scattering cross section. The projected results for the OLYMPUS experiment, as calculated by P.G. Blunden, W. Melnitchouk, and J.A. Tjon [12] are shown in Figure 3.

OLYMPUS will measure the differential scattering cross sections for electron-proton and positron-proton elastic scattering using positron and electron beams of energy $\mathrm{E}=$ 2.0 GeV from the DORIS storage ring at DESY laboratory. The electrons and positrons 


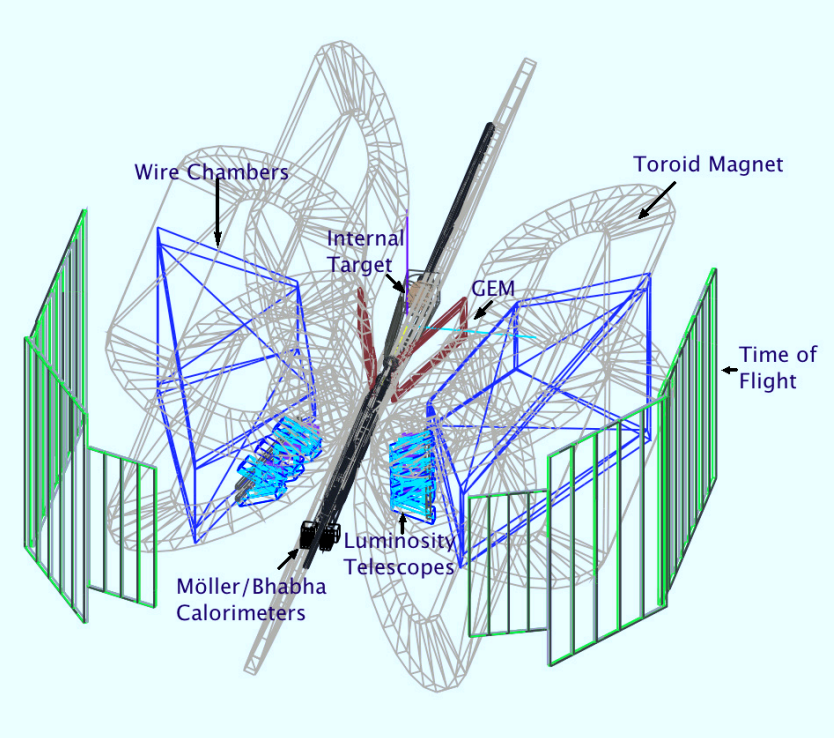

FIGURE 4: The BLAST detector with GEM and luminosity telescope tracking upgrades. The image was created using the OLYMPUS Monte Carlo.[2]

will be scattered off of an internal unpolarized hydrogen gas target and measured using the BLAST detector. The average $\mathrm{Q}^{2}$ value will be approximately $2.2 \mathrm{GeV}^{2}$ giving a deviation from unity of $\frac{\sigma_{e^{+}} p}{\sigma_{e^{-}}}$by about $6 \%$ as shown in Figure 3. [2]

\section{The BLAST detector}

The BLAST detector was originally built at MIT-Bates laboratory for polarization experiments from 2003 to 2005. It is ideal for this experiment because of its accuracy and large acceptance range. The acceptance range is from $20^{\circ}$ to $80^{\circ}$ polar angle from the beam line and $\pm 15^{\circ}$ azimuthal angle from the horizontal. The detector is left/right symmetric helping to decrease systematic errors in the data. [2] The detector is shown in Figure 4. The structure of the BLAST detector is an eight-sector toroidal magnet with most of the detector components positioned between the two horizontal sectors. The toroid magnet provides a strong almost constant magnetic field across the active detector components while dropping to zero along the beam line minimizing beam interference. The magnetic field has a maximum of $3.8 \mathrm{kG}$ and can be reversed to help minimize systematic errors. Two trapezoidal drift chambers are the main method of particle detection and tracking. These drift chambers define the acceptance range of the BLAST detector and are placed in the two horizontal sectors of the magnetic. [2] From the trajectories measured by the drift chamber in the magnetic field, particle charge and momentum can be determined and from the pulse height on the wires the particle energy can be determined. [13] This allows for a way to find the particle identification. Along the inner windows of the drift chambers are two Gas Electron Multiplier (GEM) detectors. These detectors increase the precision of the position measurements to about $50 \mu \mathrm{m}$ and allow for drift times in the drift chambers to be measured. [14] On the outside of the drift chambers are time-of-flight (TOF) scintillator detectors. These detectors 
provide additional energy measurements and an absolute "STOP" for the data and acquisition systems.

Several new detectors have been added for redundant luminosity measurements. By knowing the luminosity, the differential scattering cross section can be found to the precision needed. In addition to temperature, pressure and current monitoring there are luminosity telescopes at $12^{\circ}$ consisting of three layers of GEM detectors and multiwire-proportional chambers (MWPC) and at $1^{\circ}$ there are Møller and Bhabha luminosity monitors. [2]

\section{Target and Beam}

The target used in the OLYMPUS experiment is an internal unpolarized hydrogen gas target of "storage cell" design. In this design the target is made up of a small aluminum cell that fits into the beam line. Isotropically pure hydrogen gas is pumped into the cell, which is cooled to $35 \mathrm{~K}$. Cooling the cell lowers the conductance of the cell and increases the target thickness to $3 \times 10^{15} \mathrm{at} / \mathrm{cm}^{3}$. Using the storage cell design allows for the beam to interact with a thick target without having to pass through a window first. This increases the lifetime of the beam and allows for multiple passes. [15] The DORIS storage ring contains beams of electrons and positrons with energies up to 4.5 $\mathrm{GeV}$. OLYMPUS will be operating at an energy of $2.0 \mathrm{GeV}$ and current of $100 \mathrm{~mA}$. With this current and projected target thickness the luminosity should be $2 \times 10^{33} /\left(\mathrm{cm}^{2} \mathrm{~s}\right)$. The beams can be switched between positrons and electrons allowing for many runs to be made.[2]

\section{Timeline}

The OLYMPUS collaboration consists of 14 institutions from around the world and over 40 scientists. During summer 2010 the detector was shipped to Hamburg, Germany from MIT-Bates laboratory. The detector assembly took place from summer 2010 to summer 2011. Once assembled, the detector was rolled into place on the DORIS beam in August 2011. Detector commissioning lasts from August to December 2011 and the first official run will take place during February 2012 for four weeks. The second data taking session will take place for nine weeks from the end of October to December 2012.

Other experiments also working on measuring $\frac{\sigma_{e^{+} p}}{\sigma_{e^{-}}}$are the CLAS/PR04-116 and Novosibirsk/VEPP-3 collaborations. The CLAS collaboration used simultaneous $\mathrm{e}^{+}$ and $\mathrm{e}^{-}$beams generated from synchrotron radiation from CEBAF (continuous electron beam accelerator facility) at Jefferson Laboratory to measure the cross section ratio using the CLAS detector and an external liquid hydrogen target. Since the $\mathrm{e}^{+}$and $\mathrm{e}^{-}$ beams are generated from synchrotron radiation the experiment is not at a fixed energy. This experiment ran between December 2010 and March 2011. [16] The Novosibirsk experiment took place during 2009. The electrons and positrons were from the VEPP-3 storage ring at Novosibirsk laboratory with energy of $1.6 \mathrm{GeV}$ and were scattered on an internal hydrogen gas target. The cross section was measured with detector systems at 
$18^{\circ}$ and $64^{\circ}$.[17] The three experiments together (OLYMPUS, Novosibirsk and CLAS) should give a very accurate and complete measurement of $\frac{\sigma_{e^{+}}}{\sigma_{e^{-}}}$for a wide range of energy and $\mathrm{Q}^{2}$.

\section{SUMMARY}

The OLYMPUS collaboration will measure the multiple photon exchange contribution of elastic electron-proton scattering by measuring deviation from unity of $\frac{\sigma_{e^{+}} p}{\sigma_{e^{-}}}$as a function $\varepsilon$ within $1 \%$. To do this, electron and positron beams of $2.0 \mathrm{GeV}$ will be scattered off of an internal hydrogen gas target and the cross section will be found using the BLAST detector for an average momentum transfer of $2.2(\mathrm{GeV} / \mathrm{c})^{2}$.This will help to resolve the discrepancy between the electric to magnetic form factor ratio measurements from Rosenbluth and polarization transfer experiments.

\section{ACKNOWLEDGMENTS}

The author's research is funded by the National Science Foundation grant number NSFPHY-0969654.

\section{REFERENCES}

1. N. Kivel, and M. Vanderhaeghen, Two-photon exchange in elastic electron-proton scattering: Qcd factorization approach (2009), hep-ph/0905 0282v1.

2. D. Hasell, and et al., Olympus technical design report, Tech. rep., DESY (2010).

3. R. Bhaduri, Models of the Nucleon, Addison-Wesley, Reading, Massachusetts, 1988.

4. R. Alarcon, The olympus experiment and the proton form factor puzzle, presentation (2011), iX Latin American Symposium on Nuclear Physics and Applications.

5. M. Jones, and et al., Phys. Rev. Lett. 84, $1398-1402$ (2000).

6. M. Rosenbluth, Phys. Rev. 79, 615 - 619 (1950).

7. V. Punjabi, and et al., Phys. Rev. C 71, $1-27$ (2005).

8. A. Afanasev, S. Brodsky, C. Carlson, Y.-C. Chen, and M. Vanderhaeghen, Phys. Rev. D 72, 1-18 (2005).

9. J. T. J. Arrington, W. Melnitchouk, Phys. Rev. C 76, 1 -11 (2007).

10. G. Baym, Lectures on Quantum Mechanics, Westview Press, New York, NY, 1990, chap. 9. Potential Scattering, pp. 191-211.

11. M. Kohl, The olympus experiment at desy, presentation http://web.mit.edu/OLYMPUS/PRESENTATIONS/Kohl_DIS_20110413.pdf.

12. J. T. P.G. Blunden, W.Melnitchouk, Phys. Rev. C 72, 1 -27 (2005).

13. W. Blum, W. Reigler, and L. Rolandi, Particle Detection with Drift Chambers, Springer, Berlin, Germany, 2008.

14. F. Sauli, and M. Titov, Journal of Physics G, IOP Publishing, 2010, vol. 37, chap. 28.6.4 MicroPattern Gas Detectors, pp. 312-313, 7a edn., particle Data Group.

15. K. Zapfe, and et al., Rev. Sci. Instrum. 66, 28 (1995).

16. R. P. Bennett, Beyond the born approximation. measuring the two-photon exchange effect at clas, presentation (2011), radiative Corrections Workshop.

17. A. Gramolin, Overview of the novosibirsk experiment, presentation (2011), radiative Corrections Workshop. 